\title{
Speed, Amplitude, and Asymmetry of Lip Movement in Voluntary Puckering and Blowing Expressions: Implications for Facial Assessment
}

\author{
Karen L. Schmidt, Jessie M. Van Swearingen, \\ and Rachel M. Levenstein
}

\begin{abstract}
The context of voluntary movement during facial assessment has significant effects on the activity of facial muscles. Using automated facial analysis, we found that healthy subjects instructed to blow produced lip movements that were longer in duration and larger in amplitude than when subjects were instructed to pucker. We also determined that lip movement for puckering expressions was more asymmetric than lip movement in blowing. Differences in characteristics of lip movement were noted using facial movement analysis and were associated with the context of the movement. The impact of the instructions given for voluntary movement on the characteristics of facial movement might have important implications for assessing the capabilities and deficits of movement control in individuals with facial movement disorders. If results generalize to the clinical context, assessment of generally focused voluntary facial expressions might inadequately demonstrate the full range of facial movement capability of an individual patient.
\end{abstract}

Key Words: facial expression, Orbicularis oris, voluntary movement

Voluntary movement of the lips in puckering is important for speech, eating, drinking, and basic daily functioning of the mouth. While humans demonstrate voluntary control of lip movements, common daily facial activities (i.e., blowing, speaking, sucking, drinking, eating) take place with little conscious attention to the lip movement. The widely used Facial Action Coding System (FACS), a detailed observational rating system for facial movements related to facial expressions, defines the movements of pucker and of blowing (as if "blowing out a candle") as the contraction of the Orbicularis oris muscle (Ekman \& Friesen, 1978; Huber, 1931).

The authors are with the University of Pittsburgh, Pittsburgh PA 15260. 
The purpose of this study was to define and compare speed, amplitude, duration, and asymmetry of movement for the two expressions that we suggest differ in context and intent, but are similar in the muscle used as prime mover (O. oris). By measuring facial movement amplitude, speed, duration, and asymmetry, we sought to demonstrate potential observable differences in voluntary lip movement under conditions of a specific functionally focused movement ("blowing, as if blowing out a candle") and a general instruction to move (instructing subjects to "pucker your lips"). In addition, we investigated the relation of maximum speed and amplitude of movement for the voluntary expressions and compare the findings with similar measures previously reported for spontaneous smiles.

Significant differences in movement characteristics between facial expressions that differ in intent have been previously reported. For example, spontaneous smiles exhibiting positive emotion are less asymmetric in lip corner movement than are deliberately produced smiles (Hager \& Ekman, 1997). Spontaneous smiles of enjoyment have also been described as having a relatively short duration and as having accompanying movement of skin around the eye as compared to deliberately produced smiles (Ekman \& Friesen, 1982). Automated facial analysis of movement characteristics during spontaneous smiles indicated a consistent relation between maximum speed and amplitude in the visible upward lip corner movement of smiles. Spontaneous smiles in a solitary context (solitary spontaneous), however, exhibited smaller amplitude of movement than spontaneous smiles observed under social conditions (social spontaneous) (Schmidt, Cohn, \& Tian, 2003), despite the fact that the same muscle, Zygomaticus major, was involved in both types of smiles. Based on this example of context-dependent changes in movement control, we sought to determine whether other facial movements also differed in movement parameters under differing contexts of movement control.

Puckering and blowing expressions, two movements that also share muscle activation (O. oris), have not been studied to determine if different contexts for facial movement result in differences in the lip movement produced. In this study, we compared movement in the two voluntary expressions of the lips (O. oris), puckering and blowing, which we believe differ in intent, based on movement instructions. We predicted that the more direct and specific instructions to "blow as if blowing out a candle" provide the subjects with a functional focus that is more spontaneous once initiated and that enhances their performance of the movement. We predicted that this would result in faster speed, larger amplitude, and less asymmetric movement than puckering expressions in the same individuals. We also compared the movement in puckering and blowing, testing for a consistent relation between maximum speed and amplitude, closer to that described for spontaneous smiles.

\section{Method}

\section{Subjects}

Subjects $(\mathrm{N}=30,22$ women and 8 men) were faculty, staff, and students associated with the Department of Physical Therapy at the University of Pittsburgh. All subjects were adults over age 18 at the time of the study. 


\section{Procedure}

After obtaining informed consent for participation in the study, participants were videotaped while performing a number of common voluntary facial actions. Each subject was seated in a chair. Video recording was frontal, using a Panasonic DVX100 miniDV camcorder mounted on a tripod, recording at 30 frames per second, at a distance of approximately 5-6 feet from the subject. Head motion was minimal and was controlled for by the automated facial analysis processing method as described below (Cohn, Xiao, Moriyama, Ambadar, and Kanade, 2003). Participants were instructed to perform several facial movements. In this study we report on subject responses when asked to "pucker" and when asked to "blow, as if blowing out a candle." Both of these movements are coded as visible evidence of O. oris movement in the FACS, a detailed observational coding system for facial movement that is based on the inferred activity of individual muscles (Ekman \& Friesen, 1978). Two certified FACS coders provided consensus coding for each video sequence analyzed.

\section{Digital Video Processing and Automated Tracking of Facial Expression}

Digital video was exported to digital image format using Adobe Premier 6.5 to produce sets of 640 ( 480 pixel arrays (individual frames) with 24-bit precision for color values at a rate of 30 frames per second. In these images, each face fills the screen, approximately, with some of the faces slightly shorter or narrower in the video frame. ${ }^{1}$ Initial and final frames of the sequences were reliably coded in this study as neutral facial expressions (agreement $=100 \%$ ) and the entire sequence of images for both facial movements was analyzed (Ekman \& Friesen, 1978).

Automated Face Analysis (AFA) was used to track the movement of the lips (Cohn, Zlochower, Lien, \& Kanade, 1999). AFA is an automated computer analysis of videotape that records the movement of visible facial markers. Facial markers were located on the image of the face in the initial video frame, using the automated face detection capability of the AFA program (see Figure 1a.). The position of facial markers was reviewed by one of the authors and corrected by hand, if necessary, so that all points were directly located on the lips in the image. Facial markers in the AFA system affect the image only, they are not physically applied to the skin, unlike other motion analysis systems. A previous study of patients with facial movement disorders has established a very high reliability between the position of traditional physical facial markers on the skin and the position of facial markers in AFA. Wachtman and colleagues found a mean difference of approximately $.3 \mathrm{~mm}$ for the position of manually tracked physical facial markers as compared to AFA-tracked facial markers during several facial action tasks, with $.3 \mathrm{~mm}$ actual facial measurement corresponding to 1 pixel in the 640 ( 480 pixel

\footnotetext{
${ }^{1}$ AFA does not rely on a one-to-one correspondence to physical landmarks on the face but rather it records movement of facial features that is apparent in videotaped records. An earlier paper established the reliability of AFA-measured movement, as validated against a physical measurement system using traditional markers placed on the face. (Wachtman et al., 2001).
} 

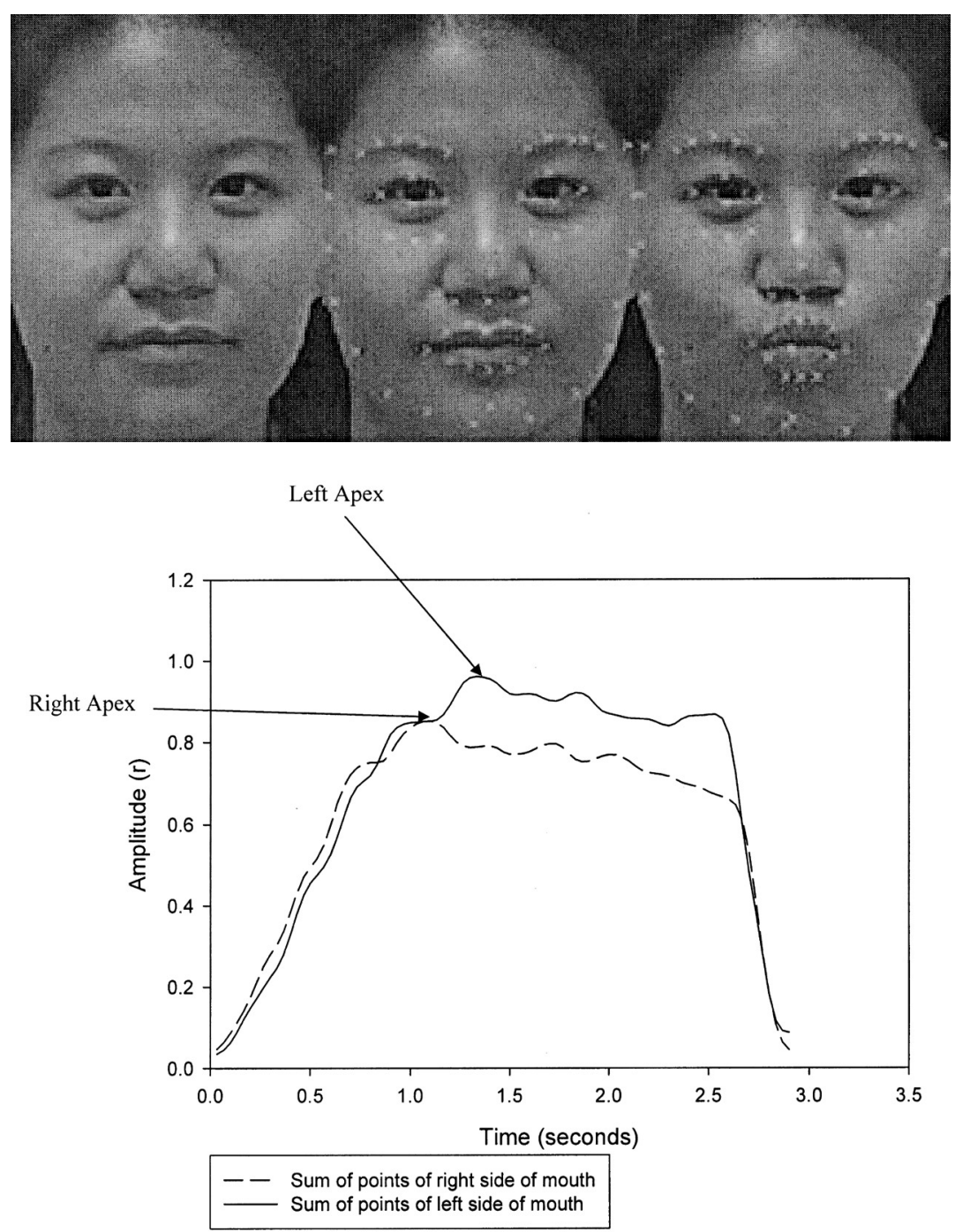

Figure 1-(a): Sample videotape frame at neutral, with facial markers, and with tracked markers at peak expression; (b) trajectory of left and right sides of the mouth in puckering [facial markers in (a) above] with movement apex marked. Values for each side represent the sum of five facial marker values at each time point.

image (Wachtman et al. 2001). Another study has established the high reliability (correlation of facial marker measurements ranging from .94 to 1.00) of movement measurements from independent trackers using the system in healthy subjects $(\mathrm{N}$ = 5) (Schmidt et al., 2003). 
The tracked facial markers that capture the movement of the lips are reported shown in Figure 1a. These markers included: both lip corners, two points on the left side of the upper lip, two points on the right side of the upper lip, two points on the left side of the lower lip, and two points on the right side of the lower lip. The center point on the upper lip and the center point on the lower lip pictured in Figure 1a were not included in these analyses; center points could not be used to calculate asymmetry, and were therefore excluded from the set of points used for analyses. As part of the automated analysis, AFA applied the Lucas-Kanade algorithm for facial marker tracking to automatically obtain $\mathrm{x}-\mathrm{y}$ coordinates for the facial markers in subsequent frames (Lien, Kanade, Cohn, \& Li, 2000). The AFA method provided head stabilization to create stabilized face images for tracking, thus separating facial movement due to expression changes from facial movement due to rigid head movements (Xiao, Kanade, \& Cohn, 2002).

Each pixel coordinate's displacement (r) from its initial position was calculated as follows:

$$
r=\sqrt{x^{2}+y^{2}}
$$

To facilitate comparison among individuals, values of $r$ were normalized. Each value was divided by the initial value for the width of the mouth from left to right lip corner at neutral expression. Because the videotape does not record a side view, the decision was made not to include AFA estimates of movement in the $\mathrm{z}$ axis in this analysis. Other analyses have confirmed the general coordination of movement in the $\mathrm{x}, \mathrm{y}$, and $\mathrm{z}$ axes over the course of facial expression (Cohn, Reed, Moriyama, Xiao, Schmidt, \& Ambadar, 2004).

\section{Data Reduction}

Proportional values of $r$ were then summed for all facial markers tracked on the left side of the mouth to create a composite variable. The same process was performed for facial markers on the right side of the mouth. Using these summed left and right variables, movement start and maximum excursion (apex) times were obtained for each side of the mouth during expression. Movement duration of the expression was operationally defined as the longest continuous increase in displacement values over the course of the expression (see Figure 1b) (Schmidt et al., 2003). Lip movements in this study generally followed the pattern shown in Figure $1 \mathrm{~b}$ with the initial movement reaching apex, with lip corners remaining in position, followed by visible movement toward resting position.

\section{Analysis}

Changes in the summed displacement values, representing movement on left and right, were analyzed to obtain values for maximum speed and amplitude of movement, as well as duration of left and right sides' movement, respectively. Maximum speed was determined by the maximum frame-to-frame difference in $\mathrm{r}$ and amplitude was determined by subtracting the value of $r$ at the apex of movement from 
the value of $r$ at initial frame of movement. Left and right values for amplitude, maximum speed, and duration of movement were averaged to produce a single dependent variable for each expression: mean speed, mean amplitude, and mean duration. Asymmetry was operationalized as the differences between left and right amplitude, left and right maximum speed, and left and right duration of movement for each of the two expressions studied. These three asymmetry variables were calculated for each expression.

Descriptive analyses were conducted for all dependent variables. A repeated measures analysis for within-subjects differences was conducted with expression (puckering or blowing) as a factor and the six variables above as outcome measures: mean maximum speed, mean amplitude, mean duration, amplitude asymmetry, maximum speed asymmetry, and asymmetry of duration. For both puckering and blowing, between-subjects analysis of the relation between maximum speed to amplitude of movement was explored with correlational analysis. The difference in correlation values for puckering and blowing was tested for significance (Rosner, 1995). This allowed for comparison between the two expressions and with previously reported results on smiles. For the between-subjects analyses of the relation of maximum speed and amplitude, overall mean values were not used and right and left movement values are reported separately for each expression, to increase comparability with previous reports. ${ }^{2}$

\section{Results}

Descriptive analysis of the primary movement measures showed mean amplitudes of .3 and .41 for puckering and blowing expressions. Maximum speeds were .075 and .084, respectively, and duration of visible movement from initial frame to apex was $.54 \mathrm{~s}$ in puckering and $.64 \mathrm{~s}$ in blowing expressions. Repeated measures analysis identified expression (pucker or blow) as a factor in within-subject differences for three of these variables (see Figure 2). Mean amplitude of movement $[F(1,29)=$ $4.44, \mathrm{p}=.04$; Figure 2, panel a] and duration of movement $[\mathrm{F}(1,29)=7.08, \mathrm{p}=$ .01 ; Figure 2 , panel c] were different in within subjects contrasts between puckering and blowing. Maximum speed asymmetry (measured as the side-to-side difference) also differed between pucker and blow expressions, with blow less asymmetric $[\mathrm{F}(1,29)=6.04, \mathrm{p}=.02$; Figure 2, panel e $]$.

To compare these voluntary expressions with spontaneous expressions previously described, we also considered the relation between maximum speed and amplitude of movement for each expression. The relation between maximum speed and amplitude on individual sides of the mouth demonstrated that pucker expressions showed a moderately strong correlation between maximum speed and amplitude of movement, for both left $(\mathrm{r}=.67)$ and right sides $(\mathrm{r}=.64)$. Maximum speed and amplitude of movement were also moderately correlated in the blow

\footnotetext{
${ }^{2}$ In an earlier paper (Schmidt et al., 2003), results were reported for a single-tracked facial marker. The possibility of error is greater for a single feature point which argues for the use of composite (average) values for left and for right-sided movement. It is possible, however, that using average values allows movement variation across points to obscure clear patterns of the relation between amplitude and maximum speed.
} 


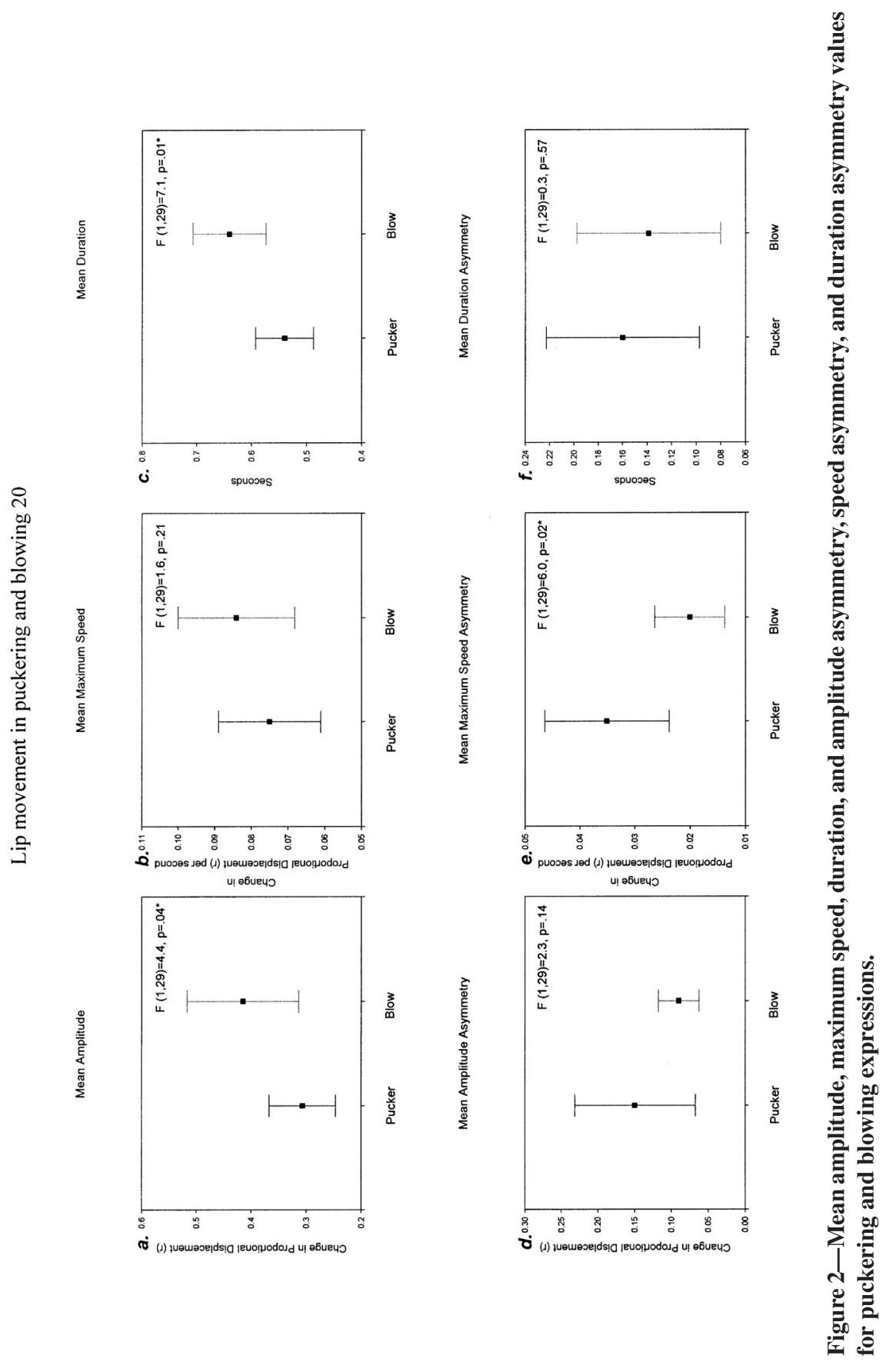


expressions, for both left $(\mathrm{r}=.67)$ and right sides $(\mathrm{r}=.55)$. The correlations do not differ between expressions for right-sided $\left(_{-}=0, \mathrm{p}=.90\right)$ or left-sided $\left({ }_{-}=0.54\right.$, $\mathrm{p}=.59$ ) movement (test for differences in correlation; Rosner 1995).

\section{Discussion}

As expected, there were differences between the two expressions within subjects. Subjects produced expressions with greater amplitude and longer duration when instructed to blow than they did when instructed to pucker. Puckering expressions were also more asymmetric than blowing expressions in terms of the maximum speed on the two sides of the mouth. Although the intent of both actions is voluntary in nature, the functional focus and possibly also the role of air flow integration with lip movement could produce both more movement and more symmetric movement of the lips in blowing. Puckering expressions, in which the instructions were focused on the movement alone, were relatively smaller and more asymmetric. The differences demonstrated between puckering and blowing are similar to differences reported for spontaneous solitary smiles as opposed to social smiles. In the case of spontaneous smiles, the solitary context where smiles were primarily smiles of amusement only produced a smaller amplitude movement on average than did the social context, with its increased communicative demands on facial movement (Schmidt et al., 2003). In this study, voluntary activation of O. oris in the context of the instruction to pucker the lips produced movement of significantly smaller amplitude than did movement with an instrumental function, blowing. As compared to spontaneous smiles, voluntary puckering and blowing expressions had very similar values for the duration of movement (smiles were $.67 \mathrm{~s}$ and $.52 \mathrm{~s}$ duration for social and solitary smiles, respectively). In terms of maximum speed, puckering and blowing (.015 and .017 , respectively ${ }^{3}$ ) were comparable to the result previously obtained for solitary and social spontaneous smiles (.006 and .016, respectively). Although voluntary in nature, the puckering and blowing expressions reported here were also produced in the social context, unlike the solitary smiles in the previous report (Schmidt et al., 2003). The amplitude of lip corner movement in spontaneous smiles in the previous study ranged from .05 for solitary smiles to .15 for social smiles, as compared to .06 and .082 for puckering and blowing reported here. ${ }^{4}$

In a between-subjects analysis of the relation of maximum speed and amplitude of movement, both the puckering and blowing expressions showed a moderately consistent relation between maximum speed and amplitude of movement, although these relations had lower values than reported for lip corner movement

\footnotetext{
${ }_{3}^{3}$ In previous studies of smiling, for example, it has been found the visible movement on the left side of the face is generally greater than that on the right (Borod, Koff, Yecker, Santschi, \& Schmidt, 1998; Pennock, Johnson, Manders, \& VanSwearingen, 1999). In addition, other reports on facial movement as measured by AFA have reported each side of the face separately (Schmidt et al., 2003).
}

\footnotetext{
${ }^{4}$ The values for puckering and blowing expressions were obtained from the sum of five facial markers and have been divided by five here to make them comparable to those for a single point as in the solitary and social smiles.
} 
in spontaneous smiles (Schmidt et al., 2003). There are not comparative values for the relation available in the literature for voluntary facial expressions, but the results support the idea that speed and amplitude relation might represent basic movement characteristics of common facial actions.

The issue of movement characteristics of facial expressions has been raised in previous work, with an emphasis on the difference in neural control of spontaneous versus deliberate expressions (Castanho \& Otta, 1999; Ekman \& Friesen, 1982; Friedman \& Miller-Herringer, 1991; Hager \& Ekman, 1997; Rinn, 1984; Schmidt et al., 2003; Shor, 1978). Although O. oris is active in forming both puckering and blowing expressions, there are differences in the quality of the movement that are more subtle and that might reveal differences between more general movement instructions, as for puckering where the intent is focused on moving the lips, and more functionally focused instructions, such as those given in this study for blowing where the intent is to move air through the lips. These differences could also have important implications for assessing facial function.

Individuals with unilateral facial paresis (the usual presentation of disorders of the facial nerve) are inherently asymmetrical. During rehabilitation to regain facial expressions, requesting the individual with unilateral facial paresis to attempt to voluntarily produce the pucker movement tends to enhance the asymmetry. The lips on the uninvolved side of the face move a greater distance into pucker and more rapidly than the lips of the involved side. The same individual with facial paresis, however, asked to attempt blowing as if to blow a stream of bubbles, produces lip movements with less asymmetry than observed for the pucker movement request (VanSwearingen, unpublished clinical observations). The intent of the request for blowing includes the function of the respiratory system, usually unaffected by the facial nerve disorder. The respiratory system response to the request for blowing might facilitate the integrated activation of muscles of both sides of the lips with the air outflow process. The result observed is greater symmetry of lip movement, with what appears to be greater speed and amplitude of movement of the lips (VanSwearingen, clinical observations).

Because of the multiple functions of daily life that involve O. oris activity to move the lips (speaking, eating, drinking, etc.), assessment of this type of movement is clinically important. The difference in function between puckering and blowing, significant for healthy individuals in this study, could be amplified in individuals with a disorder of facial movement and might have implications for facial movement rehabilitation. An assessment that relies primarily on the most directed and internally focused voluntary expressions could miss the full range of facial movement capability of an individual with a facial movement disorder. Context might not only be important for accurate assessment of facial movement abilities, but could also have a role in intervention strategies to enhance facial movement control in individuals with facial movement disorders.

\section{Acknowledgments}

This work was supported by National Institutes of Health grants MH 15279 and MH 067976 to Karen L. Schmidt. The authors acknowledge the assistance of Wen-ni Huang in the collection of data, Dr. Jeffrey Cohn and Dr. Zara Ambadar in 
providing technical assistance with AFA, and Kathleen McGinnis in the statistical analyses of this study. This research was conducted at the University Center for Social and Urban Research.

\section{References}

Borod, J.C., Koff, E., Yecker, S., Santschi,C., Schmidt, J.M. (1998). Facial asymmetry during emotional expression: Gender, valence and measurement technique. Psychophysiology, 36, 1209-1215.

Castanho, A.P., \& Otta, E. (1999). Decoding spontaneous and posed smiles of children who are visually impaired and sighted. Journal of Visual Impairment and Blindness, 93(10), 659-662.

Cohn, J.F., Reed, L.I., Moriyama, T., Xiao, J., Schmidt, K.L., and Ambadar, Z. (2004). Multimodal coordination of facial action, head rotation, and eye motion during spontaneous smiles. Proceedings of the Sixth IEEE International Conference on Automatic Face and Gesture Recognition (FGR 2004), May 17-19, 2004, Seoul, Korea, 6,129-139.

Cohn, J.F., Xiao, J., Moriyama, T., Ambadar, Z., \& Kanade, T. (2003). Automatic recognition of eye blinking in spontaneously occurring behavior. Behavioral Research Methods and Instrumental Computing, 35(3), 420-428.

Cohn, J.F., Zlochower, A., Lien, J., \& Kanade, T. (1999). Automated face analysis by feature point tracking has high concurrent validity with manual FACS coding. Psychophysiology, 36, 35-43.

Ekman, P., \& Friesen, W.V. (1978). Facial Action Coding System. Palo Alto, CA: Consulting Psychologists Press.

Ekman, P., \& Friesen, W.V. (1982). False, felt, and miserable smiles. Journal of Nonverbal Behavior, 6(4), 238-252.

Friedman, H., \& Miller-Herringer, T. (1991). Nonverbal display of emotion in public and in private: Self-monitoring, personality, and expressive cues. Journal of Personality and Social Psychology, 61(5), 766-775.

Hager, J.C., \& Ekman, P. (1997). The asymmetry of facial actions is inconsistent with models of hemispheric specialization. In P. Ekman \& E. Rosenberg (Eds.), What the Face Reveals (pp. 40-62). New York: Oxford University Press.

Huber, E. (1931). Evolution of facial musculature and facial expression. Baltimore: The Johns Hopkins Press.

Lien, J., Kanade, T., Cohn, J.F., \& Li, C.-C. (2000). Detection, tracking, and classification of subtle changes in facial expression. Journal of Robotics and Autonomous Systems, 31, 131-146.

Pennock, J.D., Johnson, P.C., Manders, E.K., VanSwearingen, J.M.(1999). Relationship between muscle activity of the frontalis and the associated brow displacement. Plastic and Reconstructive Surgery, 104, 1789.

Rinn, W.E. (1984). The neuropsychology of facial expression: A review of the neurological and psychological mechanisms for producing facial expressions. Psychological Bulletin, 95(1), 52-77.

Rosner, B. (1995). Fundamentals of Biostatistics (4th ed.). Belmont, CA: Duxbury Press.

Schmidt, K., Cohn, J., \& Tian, Y. (2003). Signal characteristics of spontaneous facial expressions: Automatic movement in solitary and social smiles. Biological Psychology, 65(1), 49-66. 
Shor, R.E. (1978). The production and judgment of smile magnitude. Journal of General Psychology, 98, 79-96.

Wachtman, G., Cohn, J., VanSwearingen, J.M., \& Manders, E.K. (2001). Automated tracking of facial features in patients with facial neuromuscular dysfunction. Plastic and Reconstructive Surgery, 107, 1124-1133.

Xiao, J., Kanade, T., \& Cohn, J. (2002). Robust full-motion recovery of head by dynamic templates and re-registration techniques. Proceedings of the IEEE International Conference on Automatic Face and Gesture Recognition, Washington, DC. 